\title{
Análisis baropodométrico de las asimetrías en los miembros inferiores en niños de 4 a 16 años: estudio observacional descriptivo
}

Baropodometric analysis of asymmetries in the lower limbs in children aged 4 to 16 years: a cross-sectional study

Carlos Dolz Peris ${ }^{1}$, Antonio Gómez Bernal ${ }^{2}$, Javier Alfaro Santafé ${ }^{2}$, Alejandro J. Almenar Arasanz ${ }^{2}$ y Francesc Boscá Muñoz ${ }^{1}$

'Fundación Universidad Católica de Valencia San Vicente Mártir. Valencia, España. ²Departamento de Formación e Investigación. Podoactiva. Huesca, España

Palabras clave:

Presión plantar, pie, postura, niños, heterometría.

\section{Resumen}

Introducción: Las heterometrías y su importancia clínica han sido un tema controvertido durante años; la literatura médica aún no ha establecido unas claras conclusiones. El presente trabajo se centra en la evaluación de las diferencias existentes en las presiones plantares y el índice de la postura del pie, entre el miembro inferior corto y largo.

Pacientes y métodos: Se trata de un estudio descriptivo, observacional y transversal, con 74 niños entre 4 y 16 años. La disimetría fue evaluada mediante pelvímetro y cinta métrica, por último, se valoró el índice de posición del pie. En cuanto al análisis baropodométrico, la carga recibida por cada pie se cuantificó mediante la plataforma de presiones T-Plate.

Resultados: Los niños con una heterometría mayor a $1 \mathrm{~cm}$ tuvieron diferencias significativas en la presión de carga recibida por cada pierna durante la estática, obteniendo un $3 \%$ de diferencia a favor de la pierna larga. Durante la dinámica hubo una diferencia de valores de $412 \mathrm{~g} / \mathrm{cm}^{2}$ en la zona del antepié de la pierna larga frente a los niños con heterometrías menores a $1 \mathrm{~cm}$; hubo significación en el FPI de la pierna larga y corta de cada uno de los estudiantes, obteniendo una presión aumentada en los casos donde se registró un FPI más alto.

Conclusiones: Los niños que presentan una heterometría mayor a un centímetro reciben mayor presión en la pierna larga. Se observaron correlaciones entre la postura del pie y la carga recibida en cada pierna, dependiendo de la presencia de la disimetría.

Keywords:

Plantar pressures, foot, posture, children, leg length inequality.
Recibido: 07-10-2019 Aceptado: $14-01-2020$

\begin{abstract}
Introduction: Heterometries and their clinical importance have been a controversial subject throughout the years; medical literature has not yet stablished any clear conclusions. The present study is focused on the evaluation of the existing differences on plantar pressures and the foot posture index, between the long and short limb.

Patients and methods: This is a descriptive, observational and transversal study, performed with 74 children with ages comprehended between 4 and 16. Dysmetries were measured with pelvimeter and measuring tape, lastly the Foot Posture Index was evaluated. In relation to the baropodometric analysis; the pressure received by each foot was quantified through the T-Plate measuring pressures' platform.

Results: Children with an heterometry bigger than $1 \mathrm{~cm}$, presented significative differences in the load pressure received by each leg during the static obtaining a $3 \%$ difference in favour of the longer leg. During the dynamic there was a difference in the obtained values of a $412 \mathrm{~g} / \mathrm{cm}^{2}$ in the forefoot zone of the longer leg compared to the children with heterometries smaller than $1 \mathrm{~cm}$, data was significative in the FPI of the longer leg than the shorter one of each student, obtaining an augmented pressure in the cases were the FPI registered was higher.
\end{abstract}

Conclusions: Students which presented a higher heterometry than $1 \mathrm{~cm}$ received a greater pressure on the longer leg. Correlations have been detected between the foot's posture and the received load on each leg depending on the dismetry. 


\section{INTRODUCCIÓN}

La diferencia de longitud en miembro inferior (DLMI) es la condición en la que ambas extremidades son notablemente diferentes ${ }^{1}$. En cuanto a datos epidemiológicos, algunos autores reflejan una prevalencia de un $40-70 \%{ }^{1}$, mientras que otros hasta un $90 \%$ de personas que sufren esta afección ${ }^{2}$. No obstante, existe la controversia en la DLMI, en el concepto, prevalencia, etiología, indicación de tratamientos, repercusiones biomecánicas y en el tiempo de intervención ${ }^{3}$. Esto se debe a distintos sesgos, tales como experiencia del clínico, demografía de los sujetos, métodos clínicos utilizados, etc. ${ }^{2}$.

Lo que está claro y documentado por los estudios efectuados es que la asimetría tiene unas consecuencias físicas negativas en las diferentes estructuras del aparato locomotor: espalda, cadera, rodilla y tobillo 4 . Esto es debido a que actúan fuerzas distintas en cada miembro y existe afectación en el patrón de la marcha, es decir, afecta a la cinética y cinemática de aquellos sujetos que presentan dicha asimetría ${ }^{4}$.

Durante la maduración ósea durante la etapa infantil debemos considerar la heterometría como un proceso de continuo cambio dinámico, donde encontraremos modificaciones hasta que el individuo alcance la madurez esquelética ${ }^{5}$. Una vez finalizada la madurez esquelética, y en ausencia de patologías, el crecimiento de las extremidades debería ser simétrico, pero en pocos casos se cumple.

Existen diversos tipos de asimetrías en los miembros inferiores, y se clasifican en dos grupos: reales y funcionales ${ }^{1,3,6}$. La dismetría estructural o real se conoce como la alteración del miembro inferior (MI), que presenta un acortamiento de un miembro respecto a otro, es decir, una diferencia en alguno de los segmentos óseos (fémur o tibia)' 1 .

Por otra parte, tenemos las asimetrías en miembros inferiores (AMI) funcionales que aparentemente a la vista son reales, pero una vez explorada, descubrimos que son el resultado de compensaciones causadas por otras alteraciones. Se producen cuando los distintos segmentos tienen la misma longitud y se observa una diferencia aparente entre los mismos. Estas afectaciones pueden ser causadas de forma multifactorial, como: básculas pélvicas, contracturas o déficits de grupos o cadenas musculares, rangos y movimientos asimétricos en las articulaciones, genu (recurvatum, varo, valgo o flexum), posicionamiento del pie asimétrico (talo, varo, valgo o equino), ángulo de marcha asimétrico, etc. ${ }^{7}$.

Se debe tener en cuenta que una diferencia de longitud aparente puede desencadenar en una estructural, ya que, según la Ley de Wolf, en las zonas óseas donde hay fuerzas de tensión se produce reabsorción ósea por los osteoclastos $y$, por el contrario, cuando existen mayores fuerzas de compresión se deposita hueso ${ }^{8}$.

El interés científico de este estudio radica en la alta incidencia de las disimetrías en la población ${ }^{2,9,10}$, y como los miembros inferiores (MMII) y el raquis compensan las mismas. Ciertos autores afirman que encontraron un aumento en la carga en del miembro más largo en pacientes con DLMI o un aumento del trabajo mecánico del miembro inferior más largo en pacientes con disimetría anatómica ${ }^{11}$. Por el contrario, autores como White y cols. ${ }^{12}$ encontraron un aumento de cargas en el miembro corto en pacientes con AMI durante una marcha. El uso de métodos de muy baja validez o no fiables para cuantificar la cantidad de acortamiento entre ambas piernas es un problema importante en la mayoría de artículos de la literatura, cuestionando así muchos de los resultados obtenidos en los estudios. Este aspecto es una de las razones que podrían explicar el porqué de los resultados confusos en la literatura.

Cuando el ser humano realiza una actividad diaria donde se ven implicados sus pies; estos ejercen una fuerza sobre la superficie subyacentey, a su vez, una fuerza de igual magnitud y dirección opuesta es ejercida sobre el pie y miembro inferior. Existen herramientas capaces de detectar y cuantificar el componente vertical de esta fuerza y la superficie de contacto que tienen las diferentes áreas del pie $\mathrm{p}^{13,14}$. Por ello, el estudio se centra en un análisis de las presiones plantares de cada pierna y cómo se distribuyen, utilizando la plataforma de presiones, pudiendo registrar datos en estática y en dinámica.

El objetivo principal de este estudio trata de evaluar las diferencias existentes en las presiones plantares que recibe en estática y dinámica el miembro inferior corto y largo en los niños con AMI.

\section{PACIENTES Y MÉTODOS}

\section{Diseño}

Se llevó a cabo un estudio descriptivo, observacional y transversal, con 74 niños entre 4 y 16 años del colegio Patronato de la Juventud Obrera (PJO), que contaban con una DLMI superior e inferior a 1 centímetro en los meses de abril y junio. El protocolo de actuación fue aprobado por el Comité Ético de la Universidad Católica de Valencia el 11 de abril de 2019. El diseño del siguiente estudio se basó y ejecutó de acuerdo con los principios de la guía Strobe ${ }^{15}$.

\section{Población de estudio}

Los datos estudiados pertenecen a una muestra compuesta por todos los escolares que acudieron los días de la toma de datos y que cumplían los siguientes requisitos: consentimiento informado de todos los participantes firmado por sus padres o tutores legales y la voluntariedad de participar en el mismo. Rigurosamente, tomamos como criterios de inclusión que los participantes tuviesen entre 4 y 16 años, que tuviesen alguna asimetría entre 0,1 y 3 centímetros, y tener la capacidad de caminar de forma independiente. De la misma forma, se excluyeron participantes que sufriesen algún tipo de dolor, que tuviesen problemas neurológicos o estructurales relevantes y que llevasen tratamiento ortésico. 
Se registraron los datos demográficos de los participantes el día de la toma de muestras, incluyendo sexo, edad, altura, peso e índice de masa corporal (IMC). Además, se midió el Foot Posture Index (FPI) descrito por Redmond ${ }^{16}$. Las variables medidas en este estudio fueron presiones plantares medidas en gramos/metro ${ }^{2}$, el FPI, las presiones plantares en porcentaje de carga y la diferencia de longitud entre ambas piernas medido en $\mathrm{cm}$ con la cinta métrica. Se tomaron tres muestras de cada medida y se obtuvo una media para disminuir el sesgo de medida.

La técnica de muestreo utilizada para este estudio fue muestreo por conveniencia; la población de estudio son 450 niños matriculados en el colegio PJO, de los cuales solo participaron 123 escolares, debido a la falta de consentimientos informados y que no hubo tiempo suficiente en el estudio para seleccionar una mayor muestra. Tras considerar los criterios de inclusión y exclusión, la población quedó finalmente constituida por 74 niños.

\section{Medición de variables}

En la presente investigación se optó por técnicas no invasivas para la medición de la heterometría. Las técnicas utilizadas fueron el pelvímetro, que es un instrumento de medición de ángulos, que nos servirá para identificar si existe una basculación a nivel pélvico y por último la medición con cinta métrica desde el ombligo a los maléolos ${ }^{17,18}$.

En primer lugar, se valoró la existencia de básculas pélvicas con el niño en carga mediante pelvímetro; seguidamente se realizó la medición en camilla mediante cinta métrica desde la fosa umbilical hasta el maléolo tibial, tras haber realizado la

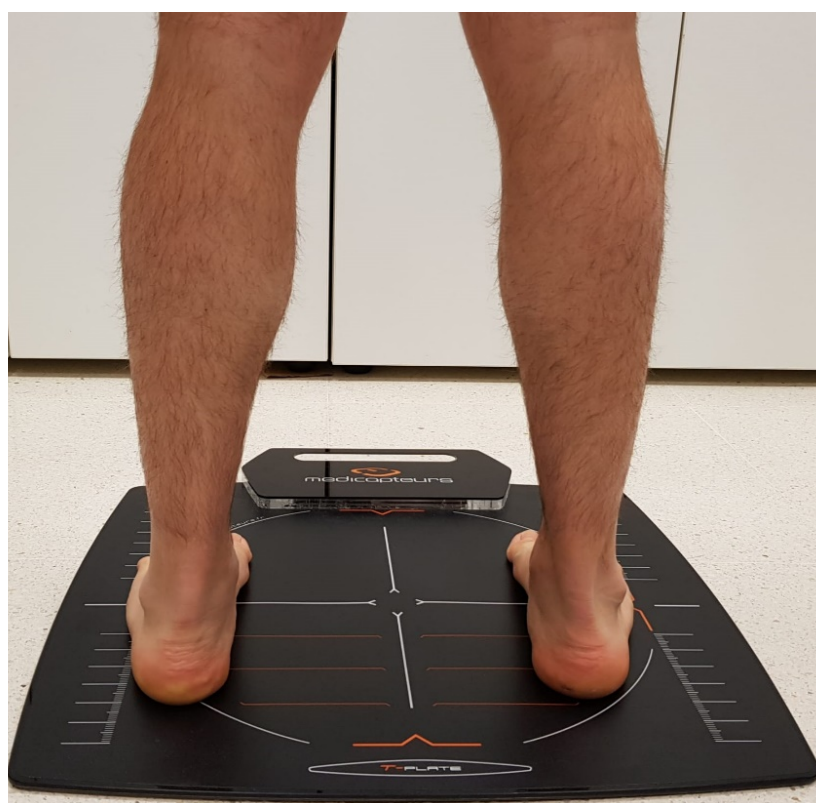

Figura 1. Prueba baropodométrica en estática. maniobra de empuje pélvico, con el fin de evitar posibles alteraciones en la posición de la cadera y pelvis. Dicha maniobra, nos ayudó a concretar si la disimetría presente era de origen estructural o funcional. Una vez medida la heterometría se dividieron en dos grupos de mayor y menor de 1 centímetro $(\mathrm{cm})$.

A continuación, con el participante en bipedestación y descalzo, se registraron los valores de FPI y, posteriormente, se tomaron los registros de presión plantar a través del software de la plataforma de presiones T-Plate (Medicapteurs, Balma, France) tanto en estática como en dinámica (Figuras 1 y 2).

En cada uno de los análisis se tomaron tres registros de cada pie, y se extrajo el promedio de cada uno de ellos para minimizar los sesgos. Durante el examen estático, se analizaron los porcentajes de carga de ambos pies; y en el examen dinámico se evaluaron por zonas (antepié, mediopié y retropié) las presiones plantares máximas.

\section{Análisis de los datos}

La distribución normal de las variables estudiadas se determinó mediante la prueba de Smirnov Kolmogorov. Para responder al objetivo principal y ver si existen diferencias significativas en la distribución de las presiones plantares se emplearon pruebas t de Student para muestras independientes con un intervalo de confianza del $95 \%$, de tal manera que se considerarán valores estadísticamente significativos aquellos cuyo valor $p$ - fuese menor de 0.05 .

Para poder cumplir el objetivo secundario de la presente investigación, se han establecido pruebas estadísticas de correlación de Pearson y pruebas paramétricas para las variables FPI y el porcentaje de presión en estática.

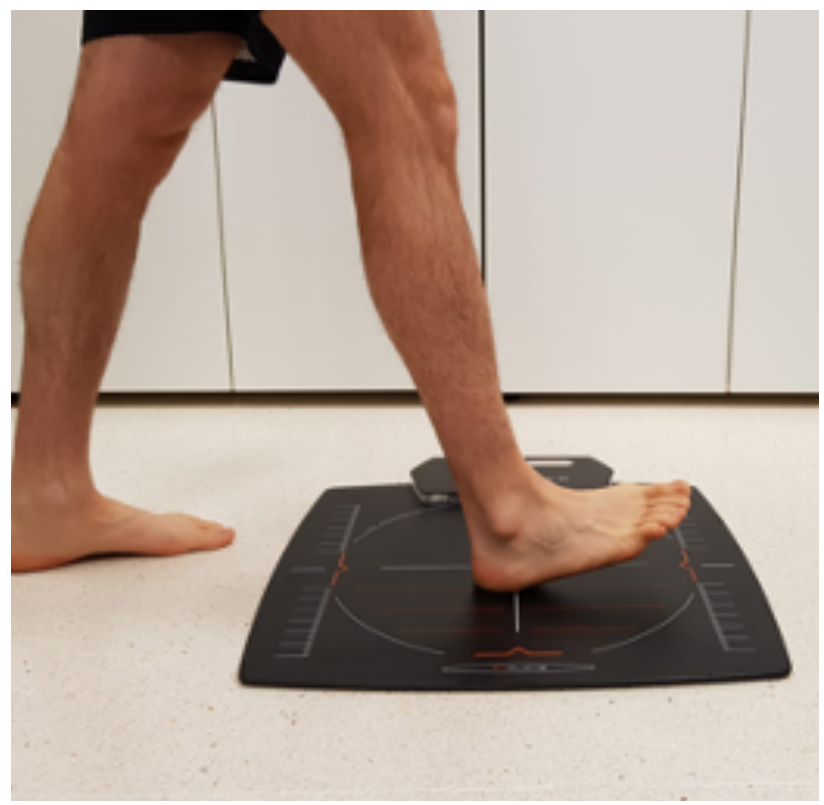

Figura 2. Prueba baropodométrica en dinámica. 


\section{RESULTADOS}

La muestra del presente estudio se compone de 74 participantes con edades comprendidas entre 4 y 16 años del colegio PJO. El grupo se clasificó en 50 sujetos con heterometrías menores a un centímetro y 24 sujetos mayores a un $\mathrm{cm}$. El resto de sujetos excluidos del estudio fue motivado por la falta de cumplimiento de los criterios de selección. Si analizamos los datos obtenidos del FPI en todos los individuos encuestados, (Tabla I) la media de la pierna larga es 6,36, mientras que en la pierna corta es de 5,58. En colegiales con heterometrías menores a un centímetro, la pierna larga marca un valor de 5,41 muy similar al de la pierna corta con 5,73. En el caso de dismetrías superiores a un centímetro la media de la pierna

Tabla I. Media y t-student de los resultados del FPI

\begin{tabular}{lccc} 
& $\begin{array}{c}\text { Pierna larga } \\
(\mathrm{FPI})\end{array}$ & $\begin{array}{c}\text { Pierna corta } \\
(\mathrm{FPI})\end{array}$ & $\begin{array}{c}\text { P-valor } \\
(<0,05)\end{array}$ \\
\hline $\begin{array}{l}>1 \mathrm{~cm} \\
\text { media }\end{array}$ & $7.28 \pm 1.31$ & $5.41 \pm 1.37$ & 0.04 \\
\hline $\begin{array}{l}<1 \mathrm{~cm} \\
\text { media }\end{array}$ & $5.73 \pm 2.85$ & $5.28 \pm 2.40$ & 0.55 \\
\hline $\begin{array}{l}\text { Todos } \\
\text { media }\end{array}$ & $6.36 \pm 2.40$ & $5.58 \pm 2.13$ & 0.05 \\
\hline
\end{tabular}

FPI: Foot Posture Index. cm: centímetro.

Tabla II. Coeficiente de Pearson variables FPI y porcentaje de carga en pierna larga. P-valor $(<0.05)$

\begin{tabular}{lll} 
Pierna larga FPI vs. estática larga $>1 \mathrm{~cm}$ & 0.24 & 0.02 \\
\hline Pierna larga FPI vs. estática larga $<1 \mathrm{~cm}$ & 0.06 & 0.11 \\
\hline Pierna pierna larga FPI vs. estática larga todos & 0.19 & 0.04 \\
\hline Pierna corta FPI vs. estática corta $>1 \mathrm{~cm}$ & -0.18 & 0.05 \\
\hline Pierna corta FPI vs. estática corta $<1 \mathrm{~cm}$ & -0.14 & 0.23 \\
\hline Pierna corta FPI vs. estática corta todos & -0.07 & 0.05
\end{tabular}

FPI: Foot Posture Index. cm: centímetro. Intervalo de confianza o significación: * $p<0,05$.

Tabla III. Media \pm desviación estándar y t-student de los valores de carga durante la estática

\begin{tabular}{lll}
$<1 \mathrm{~cm}$ & Media & P-valor \\
\hline Pierna larga (\%) & $53 \pm 5.89$ & \multirow{2}{*}{0.01} \\
\hline Pierna corta (\%) & $47 \pm 5.89$ & P-valor \\
\hline$>1 \mathrm{~cm}$ & Media & 0.60 \\
\hline Pierna larga (\%) & $49 \pm 2.31$ & P-valor \\
\hline Pierna corta (\%) & $51 \pm 2.16$ & \\
\hline TODOS & Media & 0.82 \\
\hline Pierna larga (\%) & $51 \pm 4.87$ &
\end{tabular}

Prueba T-student muestras independientes ${ }^{\star} p<0.05$. cm: centímetro. $\%$ : porcentaje. larga es de 7,28 frente a 5,41 de la pierna corta. De todos estos datos, el que resulta más valioso para el estudio es la diferencia de casi 2 puntos entre la pierna larga y corta en niños con DLMI mayor a $1 \mathrm{~cm}$. También se obtuvieron diferencias significativas una vez analizados mediante la prueba t de Student. En este caso, se observa significación en las variables del FPI entre la pierna larga y pierna corta de todos los estudiantes y de aquellos estudiantes con heterometrías superiores a $1 \mathrm{~cm}$.

Los resultados obtenidos al cotejar las variables FPI de la pierna larga-corta y los porcentajes carga en estática de la pierna larga-corta mediante la correlación de Pearson (Tabla II) indican una correlación positiva en pierna larga y negativa en pierna corta.

En la Tabla III se pueden ver los resultados obtenidos acerca de la distribución de los porcentajes de presión plantar durante la estática; tanto en la pierna larga, como en pierna corta, en todos los estudiantes con heterometrías superiores e inferiores a $1 \mathrm{~cm}$, se puede observar que existe un aumento del porcentaje de presión en la pierna larga, siendo más notorio en aquellos alumnos con asimetrías en los miembros inferiores superiores a $1 \mathrm{~cm}$. En el patrón de presiones plantares de la población de estudio se observan diferencias significativas entre el porcentaje de carga soportado por cada pierna en aquellas personas que tuvieron una heterometría superior a un centímetro.

En la Tabla IV se muestra cómo se distribuye la presión plantar en la pierna larga durante la dinámica en aquellos niños con heterometrías mayores a un centímetro frente aquellos menores a un centímetro. Se puede observar datos muy similares para la zona de retropié y mediopié y una diferencia bastante marcada en la zona del antepié con $2483 \mathrm{~g} / \mathrm{cm}^{2}$ en niños heterometrías mayores a $1 \mathrm{~cm}$, y solo $2070,88 \mathrm{~g} / \mathrm{cm}^{2}$ los que tienen menor a $1 \mathrm{~cm}$. Los valores máximos de presión plantar en la zona de antepié durante la dinámica en la pierna larga indican que existe significación entre dichas variables de presión en aquellos escolares con DLMI mayor a $1 \mathrm{~cm}$ frente a estudiantes con diferencia de miembros inferiores menor a $1 \mathrm{~cm}$, no mostrando significación en el resto de zonas.

Tabla IV. Valores máximos de presión

plantar $\left(\mathbf{g} / \mathrm{cm}^{2}\right)$ en el antepié de la pierna larga durante la dinámica

\begin{tabular}{lll} 
Antepié & Media $\left(\mathrm{g} / \mathrm{cm}^{2}\right)$ & P-valor \\
\hline DLMI $<1 \mathrm{~cm}$ & $2070.88 \pm 652.11$ & 0.01 \\
\hline DLMI $>1 \mathrm{~cm}$ & $2483.08 \pm 440.86$ & \\
\hline Mediopié & Media $\left(\mathrm{g} / \mathrm{cm}^{2}\right)$ & P-valor \\
\hline DLMI < $1 \mathrm{~cm}$ & $816.43 \pm 340.11$ & 0.16 \\
\hline DLMI $>1 \mathrm{~cm}$ & $830.43 \pm 285.86$ & P-valor \\
\hline Retropié & Media $\left(\mathrm{g} / \mathrm{cm}^{2}\right)$ & 0.27 \\
\hline DLMI < $1 \mathrm{~cm}$ & $1825.57 \pm 129.11$ & \\
\hline DLMI $>1 \mathrm{~cm}$ & $1868 \pm 237.86$ & \\
\hline
\end{tabular}

Prueba T-student muestras independientes ${ }^{*} p<0.05$. DLMI: diferencia de longitud en miembros inferiores. $\mathrm{cm}$ : centímetro. $\mathrm{g} / \mathrm{cm}^{2}$ : gramos centímetro cuadrado. 


\section{DISCUSIÓN}

En la evaluación de los datos del FPI, al observar la extremidad más larga de aquellos niños que contaban con un AMI superior a $1 \mathrm{~cm}$, los resultados indican que existe un mayor grado de pronación en comparación con la pierna corta. Por ello, en los casos donde exista un miembro inferior más largo, este se relacionará con un mayor grado de valgo y/o pronación. La razón de este aumento de valores es debido al mecanismo de compensación que se genera en la pierna larga frente a la pierna corta.

Esta información coincide con otros estudios que indican que el miembro más alto muestra un mayor grado de FPI en el $70 \%$ de los casos, es decir, un pie más pronado, entendiendo que esto ocurre como mecanismo de compensación ${ }^{19-21}$. Sin embargo, Rothbart ${ }^{22}$, en su estudio con 56 sujetos diagnosticados de DLMI mediante la maniobra de empuje pélvico, obtuvo que el miembro inferior más corto coincide con el pie con mayor grado de valgo según el FPI.

La correlación entre las variables FPI y porcentaje de carga indica una correlación positiva entre las variables FPI y porcentaje de carga de la pierna larga y corta, por ello la correlación positiva indica que a mayor grado de FPI mayor será el porcentaje de carga en la pierna larga y a menor grado de FPI menor carga recibirá la pierna corta, sobre todo en aquellos niños que cuenten con heterometrías superiores a $1 \mathrm{~cm}$. Estos resultados no se asemejan a los obtenidos por Pascual Huerta y cols. ${ }^{23}$ donde no se obtuvo relación entre las variables mediante el coeficiente de correlación de Pearson. Sin embargo, Gijon-Nogueron y cols. ${ }^{24}$ estudiaron el FPI-6 de 144 escolares y concluyeron que existe correlación estadísticamente significativa entre un pie más pronado frente a la presión máxima y superficie plantar $\left(r^{2}=0.059, p<0.001\right)$ con una predicción de $5.9 \%$.

Las presiones plantares y/o porcentaje de carga que recibía cada pierna en condiciones estáticas de todos los escolares, y al comprobar que existe significación estadística entre la carga de la pierna larga y la pierna corta (Tabla III), se observa que aquellos niños que contaban con un miembro más largo recibían mayor carga durante la estática. Estos resultados coinciden con el estudio de Mahmmod ${ }^{25}$ en el que participaron 26 pacientes con un AMI superior a $15 \mathrm{~mm}$ y donde se buscó relacionarla con la fascitis plantar. Los resultados concluyen que hay pruebas suficientes para apoyar el hecho de que la ubicación del dolor, el aumento de carga y el miembro más largo están asociados (prueba de Fisher $\mathrm{P}<0.0001$ ). Perttunen y cols. ${ }^{26}$ encontraron un aumento de la carga en el miembro más largo en pacientes con heterometrías. En otra investigación y de la misma manera Song y cols. ${ }^{27}$ detectaron un aumento en el trabajo mecánico del miembro inferior más largo en pacientes con DLMI anatómica.

Estos datos se contraponen con algunos estudios que han mostrado diferentes tipos de comportamiento mecánico entre las extremidades cortas y largas en pacientes con AMI, como por ejemplo, el estudio de Pascual Huerta y cols. ${ }^{23}$, don- de se midieron las presiones plantares en estática de sujetos con DLMI, concluyeron que no encontraron ninguna asociación en el porcentaje de presiones plantares de ambas piernas durante el análisis. Una posible razón podría ser las características del estudio, ya que la muestra se obtuvo de pacientes que sufrían algún tipo de dolor en el pie o extremidad inferior, pudiendo generar compensaciones anormales y adquiridas por el paciente. Por otro lado, White y cols. ${ }^{12}$ descubrieron un aumento de cargas en el miembro corto en pacientes con AMI y en otro estudio ${ }^{14}$ hallaron que el miembro corto recibía mayores cargas en condiciones estáticas en pacientes normales donde se simuló una diferencia de longitud en los MMII con el uso de bloques elevados.

Por último, los resultados de la dinámica indican que una diferencia de longitud en el miembro inferior mayor a $1 \mathrm{~cm}$ da como resultado una carga asimétrica de la extremidad durante la marcha. De las tres zonas analizadas, la que obtiene diferencias significativas es el antepié, por ello los niños con heterometrías reciben mayores fuerzas reactivas del suelo en esa zona. El motivo de este aumento de carga es un dato que se desconoce en este estudio, debido a que no se han realizado medidas específicas para obtener esta respuesta; aun así, se intuye que ese aumento de carga puede estar desencadenado por mecanismos compensatorios de las heterometrías en todo el miembro inferior y columna del escolar.

Estos resultados concuerdan con Perttunen y cols. ${ }^{26}$, que reclutaron a 25 pacientes con discrepancia en la longitud de las extremidades e investigaron los patrones de carga durante la marcha. La comparación bilateral indicó que discrepancias en la longitud de las extremidades producen patrones de marcha asimétricos, un aumento de las fuerzas reactivas del suelo en la fase de despegue en la extremidad larga, y a que las máximas presiones plantares se ubican en la zona del antepié de la pierna larga (más concretamente debajo de la primera cabeza). Kaufman y cols. ${ }^{28}$ investigaron a 20 sujetos entre 4 y 9 años con DLMI; el estudio concluye sosteniendo que, en general, la asimetría de estos parámetros aumenta a medida que aumenta la desigualdad en la longitud de la extremidad a partir de $2 \mathrm{~cm}$. En una revisión sistemática efectuada por Khamis y cols. ${ }^{29}$ sobre la importancia clínica de la discrepancia en la longitud de las piernas y su implicación en las desviaciones de la marcha, se encontró una relación significativa entre la AMI mayores a $1 \mathrm{~cm}$ y un mayor impacto a medida que aumenta la discrepancia produciéndose también compensaciones en el pie de la pierna larga como en toda su extremidad inferior.

Por el contrario, White y cols. ${ }^{12}$ compararon a tres grupos constituidos por niños con discrepancias anatómicas en la longitud de las piernas entre 1 y $3 \mathrm{~cm}$, a niños sin discrepancias de longitud y, por último, a niños con una discrepancia simulada de 1,30 cm en la longitud de las piernas. Los resultados indicaron que la extremidad más corta sostuvo una mayor proporción de carga y de velocidad de carga, por lo tanto finalizaron la investigación diciendo que se debe plantear el cambio de idea de que la extremidad larga recibe una mayor carga, incluso cuando las diferencias bilaterales son de hasta $3 \mathrm{~cm}$. 
El estudio presenta una serie de limitaciones, ya que no se obtuvo una muestra homogénea de todas las edades, y no se realizaron pruebas complementarias como la telemetría para cuantificar la diferencia de longitud real entre ambos miembros inferiores. Pese a ello, las pruebas clínicas seleccionadas para medir las heterometrías de los participantes han sido las de mayor evidencia científica ${ }^{17,18}$; además, el estudio cuenta con uno de los tamaños muestrales más grandes de los revisados en la literatura. Este estudio no contó con ninguna financiación externa y todos los materiales utilizados fueron prestados por la Universidad Católica de Valencia.

Como conclusión, los niños con asimetría superior a un centímetro en los miembros inferiores presentan un aumento significativo del porcentaje de carga en la pierna larga frente a la corta en condiciones de estática. Se han obtenido valores dinámicos de presión plantar más elevados en la zona del antepié en la extremidad inferior larga, no habiendo cambios significativos en el resto de zonas analizadas. Existen cambios relevantes en el FPI, habiendo una postura más pronada en el pie correspondiente a la extremidad inferior más larga. Este estudio aporta nueva información de gran interés en la práctica clínica acerca de la distribución de carga en niños con heterométricas; no obstante, futuros estudios que valoren si dichas compensaciones realizadas por los individuos pueden estar relacionadas con la capacidad de generar alguna patología específica.

\section{CONFLICTO DE INTERESES}

Los autores declaran no tener ningún conflicto de intereses relacionado con la publicación del presente artículo.

\section{FINANCIACIÓN}

Ninguna.

\section{BIBLIOGRAFÍA}

1. Gurney B. Leg length discrepancy. Gait Posture. Netherlands; 2002;15(2):195-206. DOI: 10.1016/s0966-6362(01)00148-5.

2. Knutson GA. Anatomic and functional leg-length inequality: a review and recommendation for clinical decision-making. Part I. Anatomic leglength inequality: prevalence, magnitude, effects and clinical significance. Chiropr Osteopat. 2005;13:11. DOI: 10.1186/1746-1340-13-11.

3. Brady RJ, Dean JB, Skinner TM GM. Limb Length Inequality: Clinical Implications for Assessment and Intervention. J Orthop Sport Phys Ther. 2003;33(5):221-34. DOI: 10.2519/jospt.2003.33.5.221.

4. Gibbons P, Dumper C, Gosling C. Inter-examiner and intra-examiner agreement for assessing simulated leg length inequality using palpation and observation during a standing assessment. J Osteopath Med. 2002;5(2):53-8. DOI: 10.1016/S1443-8461(02)80002-8.

5. González Herranz P. Tratamiento actual de las dismetrías y de las alteraciones rotacionales del miembro inferior. En: Conejero Casares JA, Romero Romero B, Martínez-Sahuquillo Amuedo ME, Rodríguez-Piñero Duran M. Jornadas científicas de la Sociedad Española de Rehabilitación Infantil. Sevilla: Seri; 2011. p. 38-43.

6. De Pablos J. Dismetrías de los miembros inferiores. MBA Inst. 2015; Número 13(3)

7. Rothbart BA, Estabrook L. Excessive pronation: a major biomechanical determinant in the development of chondromalacia and pelvic lists. J Manipulative Physiol Ther. 1988;11(5):373-9.
8. Jiménez Leal R. Las disimetrías (4). CPSalud (sede web)*. Madrid; (acceso 12 de febrero de 2014).

9. Brady RJ, Dean J, Skinner TM, Gross MT. Limb Length Inequality: Clinical Implications for Assessment and Intervention J Orthop Sports Phys Ther. 2003;33(5):221-34. DOI: 10.2519/jospt.2003.33.5.221.

10. Rush WA, Steiner HA. A study of lower extremity length inequality. AmJ Roentgenol Radium Ther. 1946;56(5):616-23.

11. Perttunen JR, Antilla E, Södengrad J, Merikanto J, Komi PV. Gait asymmetry in patients with limb length discrepancy. Scand J Med Sci Sports. 2004;14(1):49-56. DOI: 10.1111/j.1600-0838.2003.00307.x.

12. White SC, Gilchrist LA, Wilk BE. Asymmetric limb loading with true or simulated leg-length diferences. Clin Orthop Relat Res. 2004;(421):28792. DOI: 10.1097/01.blo.0000119460.33630.6d.

13. Zammit G V, Menz HB, Munteanu SE. Reliability of the TekScan MatScan $(R)$ system for the measurement of plantar forces and pressures during barefoot level walking in healthy adults. J Foot Ankle Res. 2010;3:11. DOI: 10.1186/1757-1146-3-11.

14. Martínez-Nova A, Sánchez-Rodríguez R, Cuevas-García JC, Sánchez-Barrado E. Estudio baropodométrico de los valores de presión plantar en pies no patológicos. Rehabilitación. 2007;41(4):155-60. DOI: 10.1016/S0048-7120(07)75509-3.

15. Von Elm E, Altman DG, Egger M, Pocock SJ, Gøtzsche C, Vandenbroucke JP, en nombre de la Iniciativa STROBE. Declaración de la Iniciativa STROBE (Strengthening the Reporting of Observational Studies in Epidemiology): directrices para la comunicación de estudios observacionales. Gac Sanit. 2008; 22(2):144-50.

16. Redmond AC, Crane YZ, Menz HB. Normative values for the Foot Posture Index. J Foot Ankle Res. 2008;1(1):6. DOI: 10.1186/1757-1146-1-6.

17. McCaw ST, Bates BT. Biomechanical implications of mild leg length inequality. BrJ Sports Med. 1991;25(1):10-3. DOI: 10.1136/bjsm.25.1.10.

18. Radcliff KE, Orozco F, Molby N, Chen E, Sidhu GS, Vaccaro AR, et al. Is pelvic obliquity related to degenerative scoliosis? Orthop Surg. 2013;5(3):171-6. DOI: 10.1111/os. 12055.

19. Blake RL, Ferguson HJ. Correlation between limb length discrepancy and asymmetrical rearfoot position. J Am Podiatr Med Assoc. 1993;83(11):625-33. DOI: 10.7547/87507315-83-11-625.

20. Lafuente B, Lafuente G. Protocolo de exploración de disimetrías. Podol clínica. 2009;10(5):140-7.

21. Blustein SM, D'Amico JC. Limb length discrepancy. Identification, clinical significance, and management. J Am Podiatr Med Assoc. 1985;75(4):2006. DOI: 10.7547/87507315-75-4-200.

22. Rothbart BA. Relationship of functional leg-length discrepancy to abnormal pronation. J Am Podiatr Med Assoc. 2006;96(6):499-504. DOI: $10.7547 / 0960499$

23. Pascual Huerta J, Arcas Lorente C, Trincado Villa L, García Carmona FJ, Fernandez Morato D. Relación entre el índice de postura del pie y presiones plantares en estática en pacientes con asimetrías de longitud menores de los miembros inferiores: estudio transversal de serie de casos. Rev Esp Podol. 2018;29(1):21-6. DOI: 10.20986/revesppod.2018.1502/2018.

24. Gijon-Nogueron G, Lopezosa-Reca E, Cervera-Marin JA, Martinez-Nova A, Sanchez-Rodriguez R, Van Alsenoy K, et al. Plantar pressure platform as clinical assesment tool in the analysis of foot posture with the foot posture index). Z Orthop Unfall. 2014;152(1):68-73. DOI: 10.1055/s0033-1360336.

25. Mahmood S, Huffman LK, Harris JG. Limb length discrepancy as a cause of plantar fascitiis. J Am Podiatr Med Assoc. 2010;100(6):452-5. DOI: 10.7547/1000452.

26. Perttunen JR, Anttila E, Södergård J, Merikanto J, Komi PV. Gait asymmetry in patients with limb length discrepancy. Scand J Med Sci Sports. 2004;14(1):49-56. DOI: 10.1111/j.1600-0838.2003.00307.x.

27. Song KM, Halliday SE, Little DG. The effect of limb-length discrepancy on gait. J Bone Joint Surg Am. 1997;79(11):1690-8. DOI: 10.2106/00004623-199711000-00011.

28. Kaufman KR, Miller LS, Sutherland DH. Gait asymmetry in patients with limb-length inequality. J Pediatr Orthop. 1996;16(2):144-50. DOI: 10.1097/00004694-199603000-00002.

29. Khamis S, Carmeli E. Relationship and signi cance of gait deviations associated with limb length discrepancy: a literature review. Gait Posture. 2017;57:115-23. DOI: 10.1016/j.gaitpost.2017.05.028. 